\title{
A simple global exercise program to overcome obesity
}

\begin{abstract}
This policy article discusses a feasible global exercise program to prevent and overcome obesity. This program demands a regular practice within a daily or every other daily schedule. Should the body be adapted to such a rhythmic regular exercise program, it becomes automatically immune against obesity. The program must not be interrupted or ceased at any time or it will drop in effectiveness.
\end{abstract}

Keywords: obesity, exercise, global program, human health
Speical Issue - 2016

\author{
Akbar Nikkhah \\ Department of Animal Sciences, University of Zanjan, Iran \\ Correspondence: Akbar Nikkhah, Department of Animal \\ Sciences, Faculty of Agricultural Sciences, University of Zanjan, \\ Chief Highly Distinguished Professor, Foremost Principal \\ Highly Distinguished Elite-Generating Scientist, National Elite \\ Foundation, Iran, Email anikkha@yahoo.com
}

Received: May 28, 2016 | Published: May 30, 2016

\section{Discussion}

Obesity is a rising problem in today's life and makes human prone to a multitude of diseases. Thus, obesity reduces life quality considerably. This epidemic problem will need to be overcome or it will ruin human life in modern ages. While prevention is a priority, obesity can be managed after incidence if such global exercise programs are practiced regularly. ${ }^{1}$

Effective exercise has two crucial components of intensity and regularity. They both must be maintained within critical limits for a global exercise program to prove successful in preventing obesity, especially in reducing abdominal and visceral fat depots. Weekly recommendations are no longer justified. Adequate physical activity must be exercised daily or at the very least every other daily. Sweating must occur and heart beating rate must effectively increase for a minimal prolonged period of $30 \mathrm{~min}$ at each exercise occasion. ${ }^{1-3}$

Continuous running even slowly meets requirements for this global exercise program. Running demands no major expenses and can be conducted almost everywhere at any time. Running favours coronary heart regions and refreshes metabolism in peripheral tissues. As a result, oxidation rate increases and waste management in gut and peripheral tissues experiences major improvements. During running, all major muscles of the body become active in energy production and consumption. As a result, waste is not accumulated and cancercausing mechanisms are interrupted and cancelled. For this cellular reason, running is considered anti-cancer. ${ }^{4-6}$ Spending only $30-60 \mathrm{~min}$ for running on a daily or every other daily basis and indeed regularly is a feasible global practice that prevents obesity and removes extra fats in abdominal areas. ${ }^{7,8}$

\section{Conclusion}

A feasible global exercise program is pursued for practice on a daily or every other daily basis. Regularity and intensity must be kept high for the exercise program to prove effective in preventing and overcoming obesity, especially in abdominal regions.

\section{Acknowledgements}

Thanked are Iran's Ministry of Science Research and Technology, and National Elite Foundation for supporting the author's global initiatives including programs of optimizing science edification in the third millennium.

\section{Conflict of interest}

The author declares no conflict of interest.

\section{References}

1. Nikkhah A. Daily Exercise: Time for a Global Anti-Obesity Willpower. Adv Obes Weight Manag Control. 2015;3(4):00063.

2. Nikkhah A. Improving Human Health through Optimizing Food Intake and Exercise Time Management: A Real-World Science. J Nutr Health Food Eng. 2015;2(5):00070.

3. Nikkhah A. A Realistic Weight Management Nutritional Regimen for Today's Life. J Nutr Health Food Eng. 2015;2(6):00077.

4. Nikkhah A. Synchronized Rhythms of Exercise and Eating: A Novel Public Program to Reduce Maternal and Pediatric Diabetes. Maternal and Pediatric Nutrition Journal. 2015;1:e101.

5. Nikkhah A. Intense Daily Physical Work to Curtail Obesity and Metabolic Pressures. Nutri Food Sci Int J. 2015;1(1):555552.

6. Nikkhah A. A Time-Efficient Exercise Formula for Normalizing Obese Body Mass Index. Adv Obes Weight Manag Control. 2015;3(2):00049.

7. Nikkhah A. Running a pragmatic anti-cancer probiotic. J Prob Health. 2016;4:e124.

8. Nikkhah A. Morning Eating and Evening Exercise: Towards an AntiCancer Lifestyle. J Cancer Prev Curr Res. 2016;4(4):00127. 